\title{
IMPULSE CONTROL FLIGHT TO THE INVARIANT MANIFOLD NEAR COLLINEAR LIBRATION POINT
}

\author{
Rufat Aminov \\ St. Petersburg State University \\ Universitetskaya nab. 7/9, St. Petersburg, \\ 199034 Russia \\ rufat.aminov@yahoo.com
}

\author{
Alexander Shmyrov \\ St. Petersburg State University \\ Universitetskaya nab. 7/9, St. Petersburg, \\ 199034 Russia \\ a.shmyrov@spbu.ru
}

\author{
Vasily Shmyrov \\ St. Petersburg State University \\ Universitetskaya nab. 7/9, St. Petersburg, \\ 199034 Russia \\ v.shmyrov@spbu.ru
}

Article history:

Received 01.08.2019, Accepted 22.09.2019

\begin{abstract}
We consider the special problem of flight from nearEarth orbit to a neighborhood of first collinear libration point of the Sun-Earth system. For such flight the numerical experiments substantiate the adequacy of the model of Hill's equations, which is the nonlinear approximation of equations of circular limited three-body problem. Otherwise, we would be obliged to use the model of limited three-body problem (or its approximation) in conjunction with the model of two-body problem for modeling of motion. During of approach to the neighborhood of libration point (in space of positions), the series of impulse controls are implemented. Controls are built on the basis of equations in variations. The purpose of implementing presented controls is hitting the manifold, where a spacecraft will be as long as possible in the linear case. This manifold is achieved when the special functions of phase variables is equal to zero. All the presented studies are illustrated in detail.
\end{abstract}

\section{Key words}

impulse control, circular restricted three-body problem, libration point, invariant manifold

\section{Introduction}

Libration points, also called Lagrange points, are particular solutions of circular limited three-body problem [13]. There are five libration points in the Earth-Sun system. It is important to note that the collinear points are unstable $\left(L_{1}, L_{2}, L_{3}\right)$ and the triangular points $\left(L_{4}\right.$, $\left.L_{5}\right)$ are stable. Libration point $L_{1}$ is at a distance of nearly 1.5 million kilometers from the center of Earth in direction to the Sun. NASA and ESA launched a series of space vehicles in the neighborhoods of $L_{1}$ and $L_{2}$, in particular, SOHO, WMAP, GENESIS, and others. The Russian company Roskosmos is planning to launch spacecraft MILLIMETRON in a neighborhood of $L_{2}$. This spacecraft is planned to be used to study various objects of the universe in millimeter and infrared ranges. In article [7] it is considered the construction of an orbit in a neighborhood of libration point $L_{2}$ for spacecraft MILLIMETRON. Also, a neighborhood of collinear libration points can be used for maneuvers in near-Earth space. There are problems in which controllable motion in a neighborhood of libration points is used in the context of counteracting a comet-asteroid hazard. Thus, the study of motion in these areas of space is relevant for modern space navigation.

\section{Formulation of problem}

Two massive bodies revolve around common barycenter in circular orbits under the influence of the Newtonian force of attraction. Also, we introduce the small third body, that attracted by two other massive bodies, but it does not have a gravitational effect on their movement. Limited three-body problem consists to describe the motion of small third body [13]. The model of Hill's equations is the nonlinear approximation of equations of circular limited three-body problem for describing of spacecraft (small body) motion in the neighborhood of collinear libration point of Earth-Sun system. In this paper, we use one variant of Hill's equations as a mathe- 


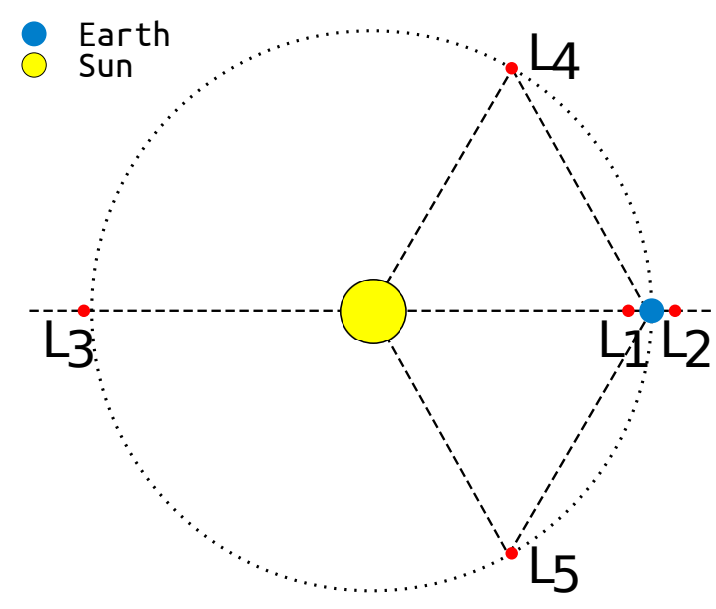

Figure 1. Location of Lagrange points

matical model of motion [11], [10]:

$$
\begin{array}{ll}
\dot{x_{1}}=y_{1}+x_{2} ; \dot{y_{1}}=-\frac{3 x_{1}}{\|x\|^{3}}+2 x_{1}+y_{2} ; \\
\dot{x_{2}}=y_{2}-x_{1} ; \dot{y_{2}}=-\frac{3 x_{2}}{\|x\|^{3}}-x_{2}-y_{1} ; \\
\dot{x_{3}}=y_{3} ; \quad \dot{y_{3}}=-\frac{3 x_{3}}{\|x\|^{3}}-x_{3} .
\end{array}
$$

Where $x=\left(x_{1}, x_{2}, x_{3}\right)$ is a spacecraft position in synodic coordinate system, $y=\left(y_{1}, y_{2}, y_{3}\right)$ are impulses, $\|\cdot\|$ is Euclidean norm. The unit of distance is nearly $1,5 \cdot 10^{6} \mathrm{~km}$, the unit of time equal $\frac{\text { year }}{2 \pi}$, the unit of speed is $303,14 \frac{\mathrm{m}}{\mathrm{s}^{2}}$ and the acceleration unit is $5,93 \cdot 10^{-5} \frac{\mathrm{m}}{\mathrm{s}^{2}}$ [11], [10].

The Hamiltonian of system (1) has the form:

$$
H=\frac{1}{2}\|y\|^{2}-\frac{3}{\|x\|}-\frac{3}{2} x_{1}^{2}+\frac{\|x\|^{2}}{2}+x_{2} y_{1}-x_{1} y_{2} .
$$

$L_{1}$ in system (1) has coordinates

$$
x^{*}=(1,0,0), \quad y^{*}=(0,1,0) .
$$

Equations (1) are obtained from the equations of the restricted circular three-body problem when we got terms of the solar potential decomposition up to second order inclusive. The idea of decomposition of solar potential was used by George William Hill in his theory of lunar motion [6]. Therefore, we call the equations (1) Hill's equations

In framework circular three-body problem the equations of uncontrollable motion of spacecraft in geocentric coordinates have the form

$$
\ddot{\tilde{x}}=-\frac{\gamma m_{1} \tilde{x}}{\|\tilde{x}\|^{3}}+\gamma m_{2}\left(\frac{R l(t)-\tilde{x}}{\|R l(t)-\tilde{x}\|^{3}}-\frac{1}{R^{2}} l(t)\right),
$$

where $\tilde{x}=\left(\tilde{x}_{1}, \tilde{x}_{2}, \tilde{x}_{3}\right)-$ geocentric coordinates of spacecraft; $\gamma-$ gravitational constant; $m_{1}, m_{2}$ - the masses of the Earth and the Sun; $R$ - the distance between the Earth and the Ssun (1 AU); $l(t)$ - a unit vector directed along the Earth-Sun line; $\|x\|=\sqrt{x x}-$ Euclidean norm.
The first and second coordinate unit vectors are located in the ecliptic plane, then

$$
l(t)=(\cos \omega t, \sin \omega t, 0),
$$

moreover, the angular velocity $\omega$ of the Earth's rotation around the Sun is determined by the formula (Kepler's third law)

$$
\omega^{2} R^{3}=\gamma\left(m_{1}+m_{2}\right)
$$

With an appropriate choice of the constant $c$, the equation (3) has the particular solution

$$
\tilde{x}^{*}(t)=\operatorname{Rcl}(t),
$$

This solution is called a rectilinear or collinear libration point. It is easy to see that the parameter $c$ satisfies the fifth order equation [6]

$$
\frac{c^{3}\left(3-3 c+c^{2}\right)}{(1-c)^{2}\left(1-c^{3}\right)}=\frac{m_{1}}{m_{2}} .
$$

For the nearest from the Earth collinear libration point $L_{1}$, we have an approximate equality

$$
c \approx\left(\frac{1}{3} \frac{m_{1}}{m_{2}}\right)^{\frac{1}{3}} \approx 0,01
$$

i.e. libration point $L_{1}$ is located at a distance of about $0.01 \mathrm{AU}$ from the Earth. This allows us to simplify the equations of motion in the neighborhood of $L_{1}$. We note $\tilde{y} \triangleq \dot{\tilde{x}}$, then the equation (3) is reduced to a hamiltonian system with Hamiltonian

$H_{1}(\tilde{x}, \tilde{y}, t)=\frac{1}{2} \tilde{y} \tilde{y}-\frac{\gamma m_{1}}{\|\tilde{x}\|}-\frac{\gamma m_{2}}{R}\left(\frac{1}{\left\|l(t)-\frac{\tilde{x}}{R}\right\|}-\frac{l(t) \tilde{x}}{R}\right)$.

Following Hill [6], we decompose the solar potential in terms of $\frac{\tilde{x}}{R}$ and reject terms higher than the second order. In this case with a nonessential constant we get

$$
H_{2}(\tilde{x}, \tilde{y}, t)=\frac{1}{2} \tilde{y} \tilde{y}-\frac{\gamma m_{1}}{\|\tilde{x}\|}-\frac{\gamma m_{2}}{R^{3}}\left(-\frac{1}{2} \tilde{x} \tilde{x}+\frac{3}{2}(l(t) \tilde{x})^{2}\right) .
$$

Further, we select the units of time and distance, so that

$$
\omega=1, \gamma m_{1}=3,
$$

i.e. 1 year equals $2 \pi$ time units. Since $\omega^{2} R^{3}=\gamma\left(m_{1}+\right.$ $\left.m_{2}\right)$, then

$$
\frac{\gamma m_{2}}{R^{3}}=\frac{m_{2}}{m_{1}+m_{2}} \approx 1
$$

and the Hamiltonian $H_{2}(\tilde{x}, \tilde{y}, t)$ takes the form

$$
H_{2}(\tilde{x}, \tilde{y}, t)=\frac{1}{2} \tilde{y} \tilde{y}-\frac{3 \tilde{x}}{\|\tilde{x}\|}-\frac{3}{2}(l(t) \tilde{x})^{2}+\frac{1}{2} \tilde{x} \tilde{x} .
$$




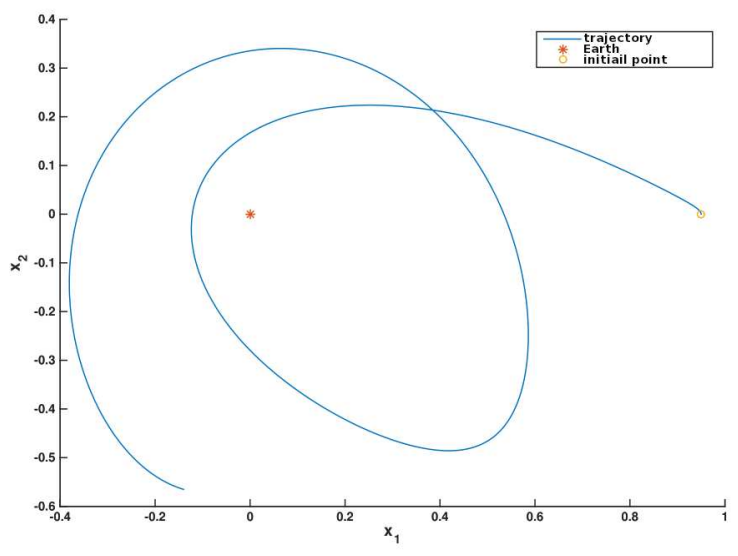

Figure 2. $x_{0}=(0.95,0.05), y_{0}=(0,1)$

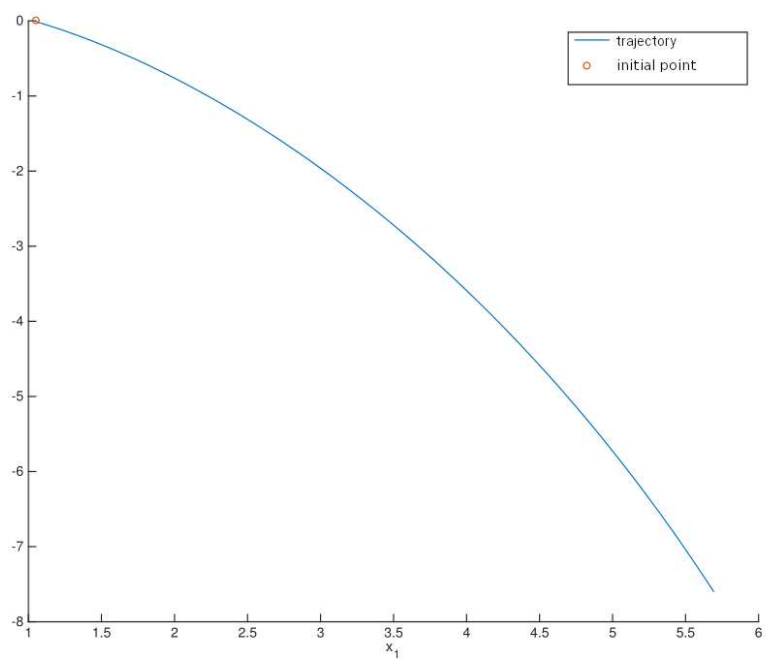

Figure 3. $x_{0}=(1.05,0.05), y=(0,1)$

Then we write the equations of motion as follows

$$
\ddot{\tilde{x}}=-\frac{3 \tilde{x}}{\|\tilde{x}\|^{3}}+3 l(t)(l(t) \tilde{x})-\tilde{x} .
$$

The equation (4) has a particular solution

$$
\tilde{x}^{* *}=l(t) .
$$

which is the libration point. Thus, the libration point of $L_{1}$ defined by the last formula is at a distance of one unit from the Earth. In the transition to the synodic coordinate system, we obtain equations (1) with Hamiltonian (2).

We linearize the system (1) in a neighborhood of libration point and obtain the linear system of equations

$$
\begin{array}{ll}
\dot{x_{1}}=y_{1}+x_{2} ; & \dot{y_{1}}=-8\left(x_{1}-1\right)+\left(y_{2}-1\right) ; \\
\dot{x_{2}}=y_{2}-x_{1} ; & \dot{y_{2}}=-4 x_{2}-y_{1} ; \\
\dot{x_{3}}=y_{3} ; & \dot{y_{3}}=-4 x_{3} .
\end{array}
$$

The characteristic equation of linear system matrix (5) is of the form the roots of this equation are the eigenvalues of the matrix of system (5)

$$
\begin{aligned}
& \lambda_{1}=\sqrt{1+2 \sqrt{7}}, \quad \lambda_{2}=-\sqrt{1+2 \sqrt{7}}, \\
& \lambda_{3}=i \sqrt{2 \sqrt{7}-1}, \lambda_{4}=-i \sqrt{2 \sqrt{7}-1} \text {, } \\
& \lambda_{5}=2 i, \quad \lambda_{6}=-2 i \text {. }
\end{aligned}
$$

Hence it is clear that a motion in ecliptic plane $\left(x_{3}=0\right.$, $y_{3}=0$ ) is unstable, because real part of eigenvalue $\lambda_{1}$ belongs right half-plane. We note that there is the singularity of linear equations (5), since the space variables $\left(x_{3}, y_{3}\right)$ are separated from variables $\left(x_{1}, x_{2}, y_{1}, y_{2}\right)$, which are describing the motion in ecliptic plane. The Figures 2 and 3 show the spacecraft departure from the small neighborhood of libration point $L_{1}$ in uncontrollable mode.

We can note several approaches to the solution of instability problem in a neighborhood of collinear point libration $L_{1}$. Usually, it can be the exit on special conditionally-periodic orbit, on which a spacecraft will be for a long time in uncontrollable mode. From the point of view of the control theory, this is the use of impulse control. There was developed the terminology for these orbits: halo orbit, a Lissajous orbit, Lyapunov orbit [4]. The other approach is the use of continuous control. In this work, we use the impulse controls, but our goal is not the exit on conditional-periodic orbit. Our goal is the exit on invariant manifold [5], on which the spacecraft will stay as long as possible in the linear case.

The promising research apparatus for such tasks is solving the optimization problem with help of gradient methods [1], [3].

There are many kinds of problem statements of maneuverings near collinear libration point. For example, the problem of station-keeping control near the halo orbit in the presence of system uncertainties [14], control strategies for formation flight of multiple spacecraft [9]. In the interesting paper [8], the authors present algorithm for holding a spacecraft on the invariant manifold near the halo orbit in the neighborhood of collinear libration point of the Earth-Moon system. In our paper, we consider a weakened boundary condition, when it is required only to hold a spacecraft in the neighborhood of $L_{1}$ for a long time.

\section{Construction of the flight trajectory}

Further we consider the motion in ecliptic plane $\left(x_{3} \equiv\right.$ $\left.0, y_{3} \equiv 0\right)$. Runge-Kutta method of the fourth order was used for the numerical simulation of a flight from a low near-Earth orbit to a neighborhood of libration point $L_{1}$. The trajectory was founded as a result of a numerical experiment with initial data

$$
x_{0}=(0.05,0.045), \quad y_{0}=(24.0834,17.4674) .
$$

In this initial data, we include the impulse control for the departure of spacecraft from the circular near-Earth

$$
\lambda^{4}-2 \lambda^{2}-27=0
$$




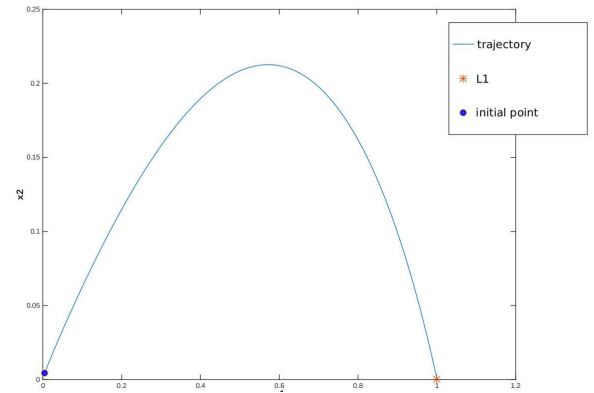

Figure 4. Trajectory of motion in the space of position

orbit. On this trajectory, a spacecraft was able to approach the small neighborhood of $L_{1}$ in positional space (Figure 4). It is important to note that the velocity assumes a sufficiently large value when spacecraft is approaching a neighborhood of libration point in space of positions. This leads to a rapid escape from the neighborhood of $L_{1}$. However, such trajectories can be used in the problem of control of spacecraft in the neighborhood of $L_{1}$.

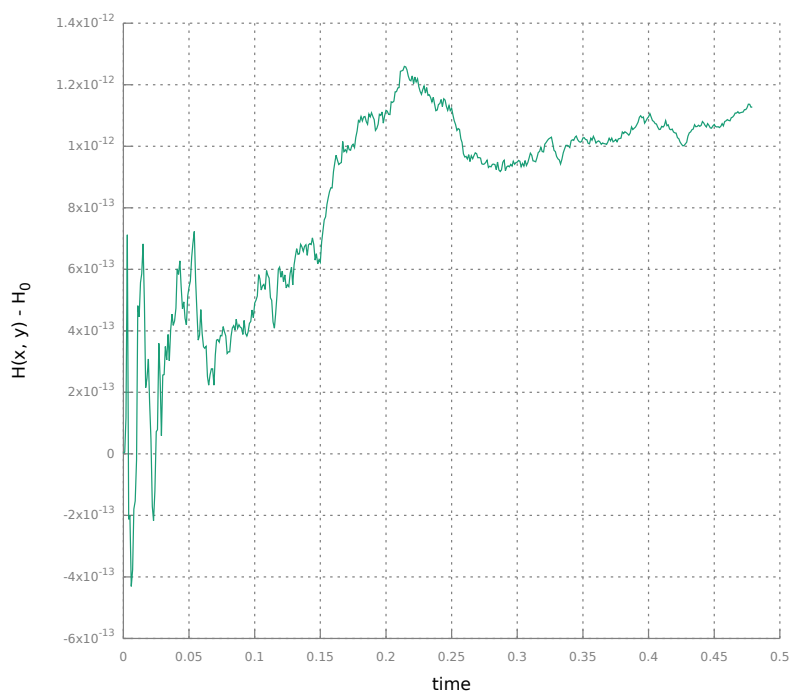

Figure 5. Hamiltonian oscillations

We use a reverse run method (calculation in reverse time, from $t=T$ to $t=0$ ) for test on the correctness of numerical results. In the absence of serious errors, when $t=0$, the result of numerical integration must coincide with $\left(x_{0}, y_{0}\right)$. We have obtained that the square root of the sum of deviations squares from initial data is about $10^{-9}$. In synodic coordinate system Hamiltonian (2) does not depend on time. Consequently, it must remain constant throughout the trajectory. The oscillations of Hamiltonian are presented in Figure 5 and amounted about $10^{-12}$. It gives a qualitative picture of the adequacy of the numerical modeling.

4 Correction of orbital motion. Problem of a long stay in a neighborhood of $L_{1}$. Equations in variations

We write the system (5) in matrix form [12]

$$
\dot{\xi}=A \xi
$$

where

$$
A=\left(\begin{array}{cc}
K & E \\
Y & K
\end{array}\right), \xi=\left(\begin{array}{c}
x_{1}-1 \\
x_{2} \\
y_{1} \\
y_{2}-1
\end{array}\right)
$$

$$
K=\left(\begin{array}{cc}
0 & 1 \\
-1 & 0
\end{array}\right), E=\left(\begin{array}{ll}
1 & 0 \\
0 & 1
\end{array}\right), Y=\left(\begin{array}{cc}
8 & 0 \\
0 & -4
\end{array}\right) .
$$

Here we consider the eigenvector-row $b_{1}$ of system (7), which corresponds to eigenvalue $\lambda_{1}$, i.e. $\lambda_{1} b_{1}=b_{1} A$. The eigenvector-rowr $b_{1}$ is determined with accuracy to the multiplier. For definiteness, we put the first element of $b_{1}$ equal to 1

$$
b_{1}=\left(1 ; \frac{\lambda_{1}^{2}-3}{\lambda_{1}\left(\lambda_{1}^{2}+5\right)} ; \frac{\lambda_{1}^{2}+3}{\lambda_{1}\left(\lambda_{1}^{2}+5\right)} ; \frac{2}{\lambda_{1}^{2}+5}\right) .
$$

We consider the linear form $d=b_{1} \xi$, so-called "hazard function" [10]. So, we have

$$
\dot{d}=b_{1} \dot{\xi}=b_{1} A \xi=\lambda_{1} b_{1} \xi=\lambda_{1} d_{1},
$$

and

$$
d(t)=d\left(t_{0}\right) e^{\lambda_{1}\left(t-t_{0}\right)} .
$$

From (8) it is clear that hazard function characterizes exponential departure from neighborhood of libration point $L_{1}$. So for long stay in a neighborhood of libration point in case of linear approximation we require

$$
d\left(t_{0}\right)=0
$$

From this equality, it is possible to find suitable changes in initial data for finding a long time staying in a neighborhood of $L_{1}$.

To achieve this goal, we can apply the apparatus of equations in variations, which is a convenient tool for analyzing a small perturbation of initial data on the solution of the system. We rewrite the system (1) in the form (for $u=0$ ):

$$
\dot{z}=f(z),
$$

where

$$
z=\left(\begin{array}{l}
x_{1} \\
x_{2} \\
y_{1} \\
y_{2}
\end{array}\right), \quad f(z)=\left(\begin{array}{c}
y_{1}+x_{2} \\
y_{2}-x_{1} \\
-\frac{3 x_{1}}{\|x\|^{3}}+2 x_{1}+y_{2} \\
-\frac{3 x_{2}}{\|x\|^{3}}-x_{2}-y_{1}
\end{array}\right) .
$$


Let there is the solution $z(t)=\phi\left(z_{0}, t_{0}, t\right)$ for uncontrolled system (9) with initial data $z\left(t_{0}\right)=z_{0}$. The system of equations in variations for (9) has the form

$$
\dot{\Phi}=\frac{\partial f}{\partial z}\left(\phi\left(z_{0}, t_{0}, t\right)\right) \Phi(t)=\left(\begin{array}{cc}
K & I \\
Y_{x} & K
\end{array}\right) \Phi(t),
$$

where [12]

$$
\begin{gathered}
Y_{x}=\left(\begin{array}{lr}
\frac{9 x_{1}^{2}}{\|x\|^{5}}-\frac{3}{\|x\|^{3}}+2 & \frac{9 x_{1} x_{2}}{\|x\|^{5}} \\
\frac{9 x_{1} x_{2}}{\|x\|^{5}} & \frac{9 x_{2}^{2}}{\|x\|^{5}}-\frac{3}{\|x\|^{3}}-1
\end{array}\right), \\
K=\left(\begin{array}{cc}
-1 & 0 \\
0 & 1
\end{array}\right), I=\left(\begin{array}{ll}
1 & 0 \\
0 & 1
\end{array}\right) .
\end{gathered}
$$

with the initial condition $\Phi\left(t_{0}\right)=E$, where $E$ is identity matrix. Further, with help of equations in variations, we study the behavior of hazard function $d(t)$, when the initial data changes from $z_{0}$ to $z_{0}+\Delta z_{0}$ (in fact, this is the implementation of impulse control).

After incrementing by phase variables at the end point of the trajectory, we have

$\phi\left(z_{0}+\Delta z_{0}, t_{0}, T\right)=\phi\left(z_{0}, t_{0}, T\right)+\Phi(T) \Delta z_{0}+o\left(\Delta z_{0}\right)$.

It is required $d(t)=0$ for staying in invariant manifold. From conditions (8) and (11) we have

$$
d(T)=b_{1}\left(\xi(T)+\Phi(T) \Delta z_{0}+o\left(\Delta z_{0}\right)\right)=0
$$

\section{Algorithm for correcting orbital motion of space- craft. Implementation of the algorithm.}

The algorithm of implementation of impulse controls for orbit correction can be described in the following form:

1. The choice of time moment $t_{0}$, in which we will change the state of the system.

2. To minimize the hazard function, we will consider $\Delta z_{0}=\left(0,0, \Delta y_{1}, \Delta y_{2}\right)$

3. Let's begin numerical integration of system (1) and simultaneously integration of system (10) up to $T$

4. As a result, we obtain a linear combination

$$
d(T)=b_{1} \xi(T)+b_{1} a_{3} \cdot \Delta y_{1}+b_{1} a_{4} \cdot \Delta y_{2}=0,
$$

where $a_{i}$ is column of matrix $\Phi(T)$ with number $i$

5. Further, we choose any $\Delta y_{1}, \Delta y_{2}$, which satisfies the last equality

In this research we use the functional for determining the increments of $\Delta y_{1}$ and $\Delta y_{2}$ in the form

$$
\Delta y_{1}^{2}+\Delta y_{2}^{2} \longrightarrow \min .
$$

The choice of the moment $t_{0}$ for the first impulse is of special interest. In this study, he was chosen heuristically and presented in Figure 6 . After the realization of impulse increments, the spacecraft is in a neighborhood of libration point of the order of 7 months. The graph of the hazard function is shown in Figure 7.

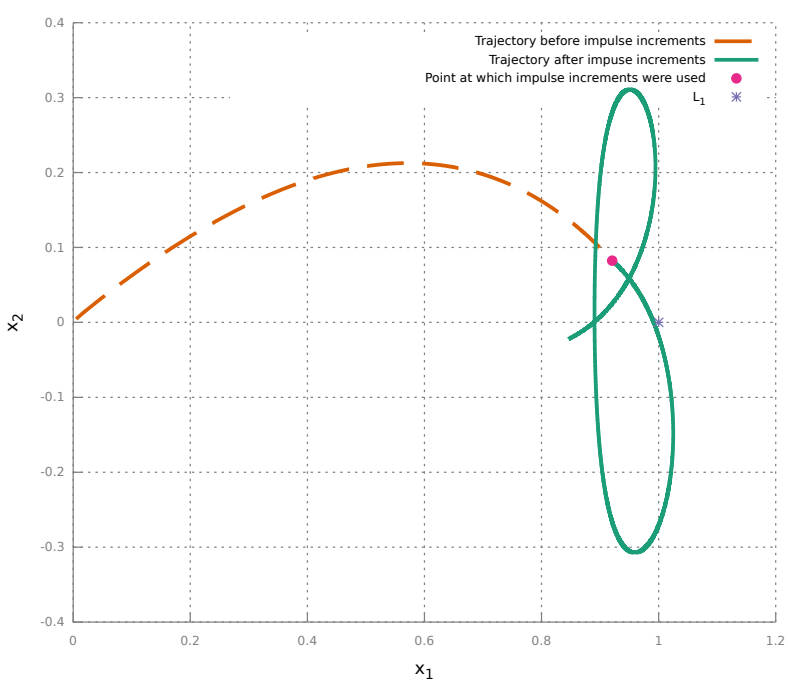

Figure 6. Realizing of impulse control at time $t_{0}=0.402531$, $\left(\Delta y_{1}, \Delta y_{2}\right)=(-0.646173,0.651189)$

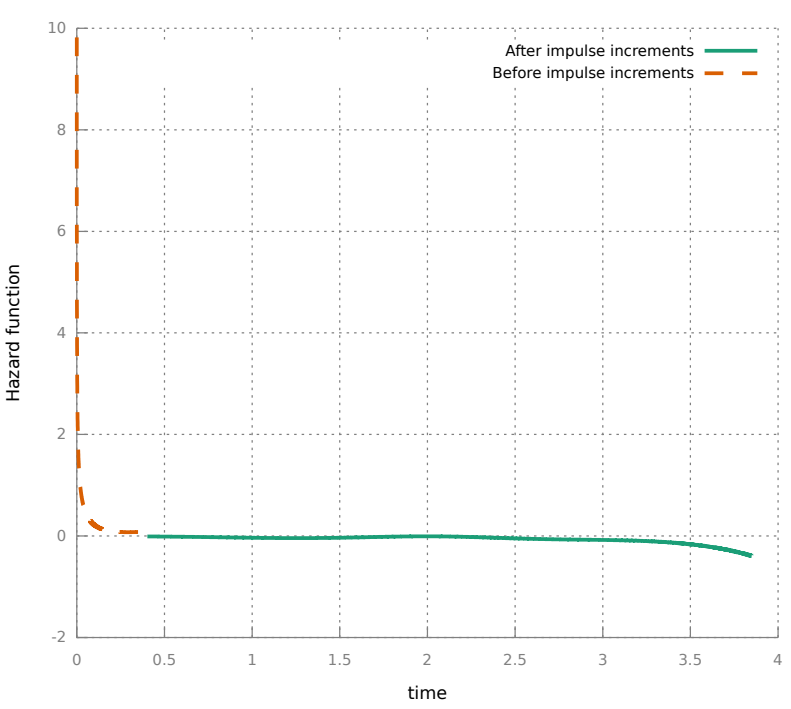

Figure 7. The value of hazard function after $t_{0}=0.402531$

For further correction of the trajectory, we can assign a number $\delta>0$. If $|d(t)|>\delta$, it is required to implement impulse control, i.e. instantaneous increment by impulses. The figure 8 shows correction of the trajectory then $|d(t)|>0.3, t>t_{0}=0.402531$ with the functional (12). The graph of hazard function is shown in Figure 9. It shows that hazard function for enough long time is in a neighborhood of zero. The spacecraft was in a neighborhood of $L_{1}$ for about 20 months. Control actions are presented in Table 1 


\begin{tabular}{lccc}
$t$ & $x_{1}$ & $x_{2}$ & $y_{1}$ \\
\hline 3.345 & 0.929411 & 0.0338744 & -0.439277 \\
7.173 & 0.865134 & -0.0172228 & -0.459914 \\
8.654 & 0.887407 & 0.047298 & -0.461464
\end{tabular}

\begin{tabular}{lccc}
$t$ & $y_{2}$ & $\Delta y_{1}$ & $\Delta y_{2}$ \\
\hline 3.345 & 0.493844 & 0.709021 & 0.382807 \\
7.173 & 0.926901 & 0.709683 & 0.383165 \\
8.654 & 0.763581 & 0.708893 & 0.382738
\end{tabular}

Table 1. The moments of time and states of system at which the impulse increments $\Delta y_{1}$ and $\Delta y_{2}$ occurred

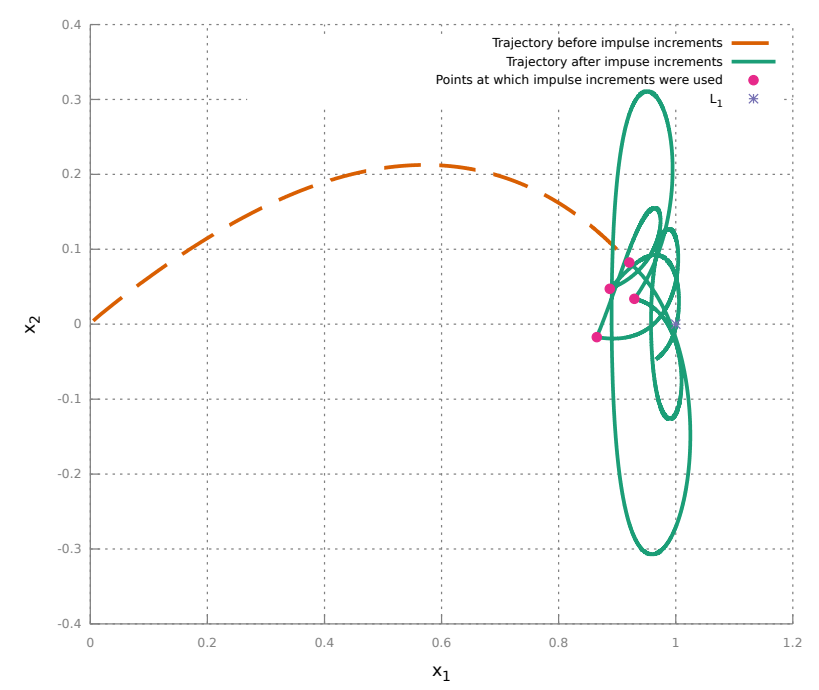

Figure 8. Controllable motion in a neighborhood of $L_{1}$

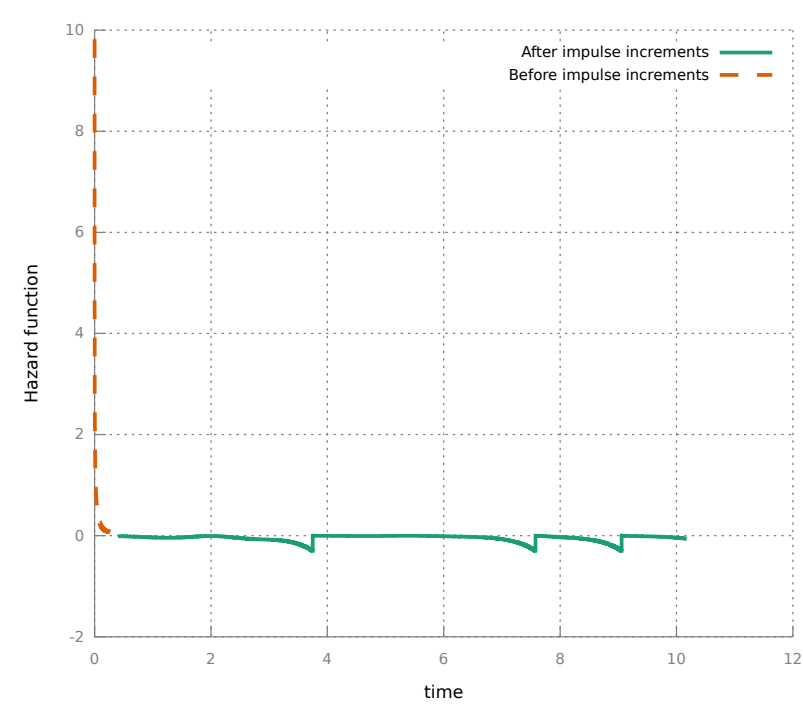

Figure 9. Values of hazard function

\section{Conclusion}

In this paper, we consider a special approach for the flight from near-Earth orbit to a neighborhood of collinear libration point. The flight path is constructed from low near-Earth orbit to a neighborhood of collinear libration point $L_{1}$ in positions space is constructed. To achieve the invariant manifold on which the spacecraft is in a neighborhood of libration point for a long time, impulse controls were constructed in the form of instantaneous increments of velocities in the ecliptic plane. To construct impulse controls, we used the apparatus of equations in variations. Further, the program was developed in $\mathrm{C}++$. In automatic mode, this program builds impulse controls that are optimal in cost, when spacecraft leaves the invariant manifold. With the help of this program, we realized the numerical simulation, in which it was possible to keep the spacecraft in a neighborhood of libration point $L_{1}$ on time interval of the order of 1.5 years with the implementation of three impulse controls.

Further development of this research is possible in the synthesis of different approaches for a long stay in a neighborhood of libration point.

\section{References}

Bazhanov P., Kotina E., Ovsyannikov D., and Ploskikh V. Optimization algorithm of the velocity field determining in image processing Cybernetics and Physics, 7 (4), 2018. P. 174-181.

Farquhar R.W. The control and use of libration-point satellites: Ph.D. Dissertation, Stanford University, Stanford, 1968.

Golovkina A., Ovsyannikov D., and Olaru S. Performance optimization of radioactive waste transmutation in accelerator driven system Cybernetics and Physics, 7 (4), 2018. P. 210-215.

Gomez G., Llibre J., Martinez R., and Simo C. Dynamics and mission design near libration points. Vol. 1. Fundamentals: The case of collinear libration points, World Scientific Publishing, Singapore, New Jersey, London, Hong Kong, 2001.

Guckenheimer J., and Holmes P. Nonlinear Oscillations, Dynamical Systems, and Bifurcations of Vector Fields, Springer-Verlag, New York, 1983.

Hill G.W. Researches in the lunar theory, American Journal of Mathematics, 1878, 1, P. 5-26, 129-147, 245260.

Ilyin I.S. Selection of a nominal orbit for the Millimetron spacecraft from the periodic orbits family in the vicinity of the Sun-Earth $L_{2}$ libration point, KIAM Preprint, 46, Moscow, 2013.

Lian Y., Gomez G., Masdemont J.J., and Tang G. Station-keeping of real Earth-Moon libration point orbits using discrete-time sliding mode control, Communications in Nonlinear Science and Numerical Simulation, 2014, 19(10), P. 3792-3807.

Marchand B.G., and Howell K.C. Control strategies for formation flight in the vicinity of the libration points, Journal of Guidance Control and Dynamics, 2005, 28(6), P. 1210-1219.

Shmyrov A., and Shmyrov V. The optimal stabilization of orbital motion in a neighborhood of collinear 
libration point, Numerical Algebra, Control \& Optimization, 2017, 7(2), P. 185-189.

Shmyrov V.A. Stabilization of the controlled orbital movement of a space vehicle in the neighbourhood of collinear libration point $L_{1}$, Vestnik SanktPeterburgskogo universiteta. Seriya 10: Prikladnaya matematika. Informatika. Protsessy upravleniya 2005. is. $1-2$, P. 192-198.

Shymanchuk D.V., Shmyrov A.S. Construction of the return trajectory to the neighborhood of the collinear libration point of the Sun-Earth system, // Vestnik
Sankt-Peterburgskogo universiteta. Seriya 10: Prikladnaya matematika. Informatika. Protsessy upravleniya. is. 2 , 2013. P. 75-84.

Szebehely V. Theory of orbit - The restricted problem of three bodies. Academic Press, New York, 1967.

Zhu M., Karimi H.R., Zhang H., Gao Q., and Wang Y. Active Disturbance Rejection Station-Keeping Control of Unstable Orbits around Collinear Libration Points, Mathematical Problems in Engineering, 2014, art. 410989. 